\title{
Lectin-based proteomic profiling of aged skeletal muscle: Decreased pyruvate kinase isozyme M1 exhibits drastically increased levels of $N$-glycosylation
}

\author{
Kathleen O'Connell, Philip Doran, Joan Gannon, Kay Ohlendieck* \\ Department of Biology, National University of Ireland, Maynooth, Co. Kildare, Ireland
}

Received 5 March 2008; received in revised form 10 April 2008; accepted 21 April 2008

\begin{abstract}
Since various neuromuscular diseases are associated with abnormal glycosylation, it was of interest to determine whether this key post-translational modification is also altered in aged skeletal muscle. Lectins represent highly versatile carbohydrate-binding proteins that are routinely employed for the characterization of glycoproteins. Here, we used the lectin wheat germ agglutinin (WGA) for the proteomic profiling of senescent fibers. WGA labeling of the soluble proteome from 3-month- versus 30-month-old rat gastrocnemius muscle, following two-dimensional gel electrophoretic separation, resulted in the identification of 13 distinct protein species. Analysis of WGA binding levels, in conjunction with mass spectrometric fingerprinting, revealed that one isoform of a major metabolic muscle protein exhibited a drastic alteration in the content of sialic acid and $\mathrm{N}$-acetylglucosaminyl sugar residues. Pyruvate kinase isoform M1 with protein accession number gi|16757994|, exhibiting a pI of 6.6 and an apparent molecular mass of $57.8 \mathrm{kDa}$, showed a six fold increase in $\mathrm{N}$-glycosylation and a three fold decrease in protein expression. In contrast to comparable levels of $\mathrm{N}$-glycosylated proteins in young adult versus senescent muscle, as judged by fluoresceinconjugated WGA labeling of transverse muscle cryosections, staining with antibodies to the M1 isoform of pyruvate kinase showed reduced expression of this cytosolic element. Furthermore, activity assays demonstrated a reduced activity of this glycolytic enzyme in senescent muscle. This agrees with the idea that abnormal post-translational modifications in key metabolic enzymes may be involved in the conversion of aged muscle to slower twitch patterns and a drastic shift to more aerobic-oxidative metabolism.
\end{abstract}

(C) 2008 Elsevier GmbH. All rights reserved.

Keywords: Aging; Glycoproteome; Lectin; Wheat germ agglutinin; Pyruvate kinase; Sarcopenia

\section{Introduction}

The evolutionary role of glycans in nature and their enormous structural and functional diversity is currently under intense investigation (Varki, 2006). Glycosylation

\footnotetext{
*Corresponding author. Tel.: + 35317083842 ; fax: +35317083845 .

E-mail address: kay.ohlendieck@nuim.ie (K. Ohlendieck).
}

is one of the most frequent post-translational modifications found in proteins and plays a central role in cellular mechanisms of health and disease (Ohtsubo and Marth, 2006). Oligosaccharide attachment represents a common protein modification that influences the folding of the nascent peptide chain and stability of glycoproteins, modifies enzymatic activity, controls protein secretion events, presents critical information about the cellular targeting of a newly synthesized protein, and 
provides specific recognition motifs for other proteins in cell-cell interactions (Gagneux and Varki, 1999; Freeze, 2006). In contrast to the glycosaminoglycans of proteoglycans, the carbohydrate moieties of a typical glycoprotein are smaller and more structurally diverse (Ohtsubo and Marth, 2006). The two main types of carbohydrate-protein conjugation in glycoproteins are $N$-glycosylation (Yan and Lennarz, 2005) and $O$-glycosylation (Peter-Katalinic, 2005). O-linked carbohydrate bonding occurs through serine or threonine, and $N$-glycosidic linkage to asparagine residues (Ohtsubo and Marth, 2006).

A variety of biochemical methods have been employed to study glycan modifications, especially involving mass spectrometric technology (Budnik et al., 2006; Bond and Kohler, 2007; Morelle and Michalski, 2007). Over the last few years, mass spectrometry-based glycoproteomics has established itself as an important analytical instrument for studying normal and pathological aspects of glycoproteins in a global way (Harvey, 2005; Geyer and Geyer, 2006; Wuhrer et al., 2007). An essential tool for the specific recognition of sugars has been the lectin class of carbohydrate-binding proteins (Cummings, 1994). Lectins interact with complex carbohydrates in a non-covalent manner that is usually both reversible and highly specific (Ambrosi et al., 2005; Naeem et al., 2007). That lectin-carbohydrate interactions can also be exploited in modern proteomic applications has been shown by numerous studies (Qiu and Regnier, 2005; Ohtsu et al., 2005; Durham and Regnier, 2006). Recently, Schwientek et al. (2007) have utilized serial lectin affinity chromatography in combination with two-dimensional gel electrophoresis and matrix-assisted laser desorption/ionization time-offlight (MALDI-ToF) mass spectrometry in an innovative glycoproteomic approach, showing the usefulness of lectin binding properties as analytical tools in highthroughput glycobiological studies. Here, we have used one of the most commonly employed lectin in glycobiology, wheat germ agglutinin (WGA), which selectively recognizes sialic acid and $N$-acetylglucosaminyl sugar residues (Bhavanandan and Katlic, 1979; Bains et al., 1992; Muraki et al., 2002).

Various muscle diseases are associated with abnormal protein glycosylation (Martin-Rendon and Blake, 2003; Barresi et al., 2004; Haliloglu and Topaloglu, 2004). Defects in the glycosylation of alpha-dystroglycan, an extracellular member of the sarcolemma-spanning dystrophin-glycoprotein complex, are linked to various forms of muscular dystrophy (Brockington and Muntoni, 2005). In analogy, since muscle aging is also associated with fiber degeneration (Edstrom et al., 2007), it was of interest to conduct a global glycoprotein survey of age-related muscle wasting using peroxidaseconjugated WGA. The age-dependent decline in skeletal muscle mass and function, now generally referred to as sarcopenia of old age (Doherty, 2003), is a major factor in blocking healthy aging (Greenlund and Nair, 2003). Sarcopenia is believed to be due to a multi-factorial pathology (Navarro et al., 2001) and contributes to increased physical disability, frailty and loss of independence in the elderly (Janssen et al., 2002). Proteomics has been widely employed in the biochemical studies of aging-induced alterations in muscle fibers, as reviewed by Sharov and Schoneich (2007). Recent proteomic profiling studies of sarcopenia revealed a drastically perturbed protein expression pattern in aged fibers involving elements of the contractile apparatus, metabolism and stress response (Piec et al., 2005; Gelfi et al., 2006; O'Connell et al., 2007; Doran et al., 2008). Altered post-translational modifications were shown to play a key role in biological aging (Schoneich et al., 1999). For example, altered tyrosine nitration was found in key muscle proteins, such as the sarcoplasmic reticulum $\mathrm{Ca}^{2+}$-ATPase, enolase, aldolase, creatine kinase, tropomyosin, glyceraldehyde-3-phosphate dehydrogenase, myosin light chain, pyruvate kinase, actinin, actin, and the ryanodine receptor (Kanski et al., 2003, 2005).

Building on these findings, we have conducted here a comparison of young adult versus senescent skeletal muscle. As an established tissue model of sarcopenia, 30-month-old rat gastrocnemius muscle was used (Edstrom et al., 2007; Doran et al., 2007a). WGA labeling revealed an altered glycosylation in the major metabolic muscle protein pyruvate kinase and a concomitant reduction in enzyme activity. Besides the key enzymatic reactions provided by hexokinase and phosphofructokinase, the trans-phosphorylation from phosphoenolpyruvate and ADP to pyruvate and ATP represents the final rate-limiting step of glycolysis (Munoz and Ponce, 2003), making reduced pyruvate kinase activity in aged fibers an important finding. These results indicate that sarcopenia is associated with drastic alterations in the expression, glycosylation and activity of a key metabolic enzyme in skeletal muscle, which agrees with the notion that senescent muscles undergo a shift to a slower-twitching phenotype with a more aerobic-oxidative metabolism (Deschenes, 2004).

\section{Materials and methods}

\section{Materials}

Peroxidase-conjuagted WGA and Ulex europaeus agglutinin-1 (UEA-1) were purchased from EY Laboratories (San Mateo, CA, USA) and its competitive sugars, $N$-acetylglucoseamine and fucose, were from Sigma Chemical Company (Dorset, UK). Protease inhibitors were obtained from Roche Diagnostics (Mannheim, Germany). Ultrapure Protogel acrylamide 
stock solutions were from National Diagnostics (Atlanta, GA, USA). The 2-D Quant kit for protein quantification, isoelectric focusing $\mathrm{pH}$ gradient (IPG) drystrips, ampholytes, acetonitrile and electrophoretic cover fluid were purchased from Amersham Bioscience/GE Healthcare (Little Chalfont, Bucks, UK). Sequencing grade-modified trypsin was from Promega (Madison, WI, USA). For MALDI-ToF mass spectrometry, the external calibrant PeptideMix-1 and the matrix $\alpha$-cyano-4-hydroxycinnamic acid were obtained from LaserBio Labs (Sophia-Antipolis, France). For blotting experiments, nitrocellulose membranes were obtained from Millipore (Bedford, MA, USA), chemiluminescence substrate from Pierce and Warriner (Chester, UK) and X-ray film from Fuji Photo Film Co. Ltd. (Tokyo, Japan), as well as Ponceau S-Red staining solution, GBX developer, GBX fixer and GBX replenisher from Sigma Chemical Company. Primary antibodies for Western blotting and immunofluorescence microscopy were purchased from Abcam Plc., Cambridge, UK (antibodies ab6191 and ab38237 to pyruvate kinase) and Novacastra Laboratories, Newcastle upon Tyne, UK (antibody NCL-b-DG to $\beta$-dystroglycan). Secondary peroxidase-conjugated and fluorescent antibodies were obtained from Chemicon International (Temecula, CA) and Molecular Probes (Eugene, Oregon, USA), respectively. For microscopy, Superfrost Plus positively charged microscope slides were purchased from Menzel Gläser (Braunschweig, Germany), optimum cutting temperature (OCT) compound was from Sakura Finetek Europe B.V. (Zoeterwoude, the Netherlands), $p$-phenylenediamine (PPD) glycerol from Citifluor Ltd. (London, UK), and diamidino-phenylindole (DAPI) and the hydrophobic liquid blocker PAP pen from Sigma Chemical Company. All other chemicals were of analytical grade and obtained from Sigma Chemical Company.

\section{Preparation of crude skeletal muscle protein complement}

As an established model system of sarcopenia, 30-month-old gastrocnemius muscles from an outbred strain of albino Wistar rats were used. For comparative studies, cohorts of senescent animals and 3-month-old control rats were kept at a standard light-dark cycle and fed ad libitum in the Animal Facility of the Department of Physiology, Trinity College, Dublin, as previously described (Doran et al., 2007b). Fresh muscles were dissected, and then snap-frozen for biochemical or cell biological analysis in liquid nitrogen or liquid nitrogencooled isopentane, respectively, and then stored at $-70{ }^{\circ} \mathrm{C}$ until further usage. Muscle specimens were kept frozen in liquid nitrogen while being pulverized using a mortar and pestle. For enzymatic assays, equal quantities of $100 \mathrm{mg}$ wet weight of muscle were used for both 3-month- and 30-month-old samples. The resulting powder was placed into $1 \mathrm{ml}$ of homogenization buffer (0.5 M HEPES, $\mathrm{pH} 7.4,200 \mathrm{mM}$ EGTA, 10\% (w/v) sucrose, $3 \mathrm{mM} \mathrm{MgCl} 2,0.1 \%$ (w/v) $\mathrm{NaN}_{3}$ ). In addition, to prevent proteolytic degradation, the extraction buffer was supplemented with a protease inhibitor cocktail containing $1 \mu \mathrm{M}$ leupeptin, $1.4 \mu \mathrm{M}$ pepstatin, $0.15 \mu \mathrm{M}$ aprotinin, $0.5 \mathrm{mM}$ soybean trypsin inhibitor, $0.2 \mathrm{mM}$ pefabloc, $0.3 \mu \mathrm{M}$ E-64, and $1 \mathrm{mM}$ EDTA (Doran et al., 2004). Suspensions were sonicated for $10 \mathrm{~s}$ with a Sonoplus HD 2200 apparatus from Bandelin (Berlin, Germany) and then incubated on ice with vortexing every $10 \mathrm{~min}$ for $10 \mathrm{~s}$, for $4 \mathrm{~h}$. Samples were centrifuged for $20 \mathrm{~min}$ at $20,000 \mathrm{~g}$ (5417R centrifuge; Eppendorf, Hamburg, Germany) and the amount of protein was quantified using the Amersham 2-D Quant kit as per the manufacturer's instructions. Pyruvate kinase activity was measured according to the enzymatic method of Bergmeyer and Bernt (1974) using a coupled lactate dehydrogenase system, whereby the disappearance of NADH was monitored at $\lambda=340 \mathrm{~nm}$ using a Cary Eclipse fluorescence spectrophotometer (Varian Inc., CA, USA) over a period of 5 min per assay. For twodimensional gel electrophoretic separation, muscle specimens were pulverized as described above but resuspended in $1 \mathrm{ml}$ of lysis buffer (7 M urea, $2 \mathrm{M}$ thiourea, $20 \mathrm{mM}$ Tris, $65 \mathrm{mM}$ CHAPS, $100 \mathrm{mM}$ dithiothreitol and $5 \%$ (v/v) ampholytes). Samples were homogenized for $10 \mathrm{~s}$ each with a model 1200 Polytron microhomogenizer (Kinematica AG, Switzerland). To decrease viscosity of the suspensions, $2 \mu \mathrm{l}$ DNase-1 (200 units) was added per $100 \mu 1$ lysis buffer, as well as the above-described protease inhibitor cocktail. Samples were incubated at room temperature for $3 \mathrm{~h}$ on a shaker with vortexing for $10 \mathrm{~s}$ every $10 \mathrm{~min}$. The suspension was then centrifuged at $20,000 \mathrm{~g}$ for $20 \mathrm{~min}$ and the lower protein-containing layer of the supernatant was used for isoelectric focusing.

\section{Gel electrophoretic separation of the skeletal muscle proteome}

In preparation for isoelectric focusing, IPG strips were rehydrated for $12 \mathrm{~h}$ with rehydration buffer (7 M urea, $2 \mathrm{M}$ thiourea, $10 \mathrm{mM}$ Tris, $65 \mathrm{mM}$ CHAPS, $100 \mathrm{mM}$ DTT and 5\% (v/v) pH 3-10 ampholytes; containing $0.05 \%(\mathrm{w} / \mathrm{v})$ bromophenol blue as tracking dye) in a re-swelling tray from Amersham Biosciences/ GE Healthcare. Rehydration of 24-cm strips was achieved with $450 \mu 1$ rehydration buffer and, after re-swelling, the IPG strips were loaded gel-side-up in an Amersham Ettan IPGphor manifold and covered with $108 \mathrm{ml}$ cover fluid. An appropriate amount of sample was added by anodic cup loading to the strips 
and run on an Amersham IPGphor IEF system, with the following running conditions: $30 \mathrm{~V}$ for $1 \mathrm{~h}, 100 \mathrm{~V}$ for $1 \mathrm{~h}$, $1000 \mathrm{~V}$ for $1 \mathrm{~h}, 6000 \mathrm{~V}$ for $1 \mathrm{~h}, 8000 \mathrm{~V}$ for $3 \mathrm{~h}, 500 \mathrm{~V}$ for $4 \mathrm{~h}$, and finally $8000 \mathrm{~V}$ for $3 \mathrm{~h}$. Following isoelectric focusing, IPG strips were equilibrated twice for $20 \mathrm{~min}$ using $6 \mathrm{M}$ urea, 30\% (w/v) glycerol, $2 \%(\mathrm{w} / \mathrm{v})$ SDS, $100 \mathrm{mM}$ Tris- $\mathrm{HCl}, \mathrm{pH} 8.8$, whereby the first incubation step was performed with the addition of $100 \mathrm{mM}$ DTT and the second incubation step with $0.25 \mathrm{M}$ iodoacetamide. The strips were then briefly washed in SDS running buffer $(0.125 \mathrm{M}$ Tris, $0.96 \mathrm{M}$ glycine, $0.1 \%$ $(\mathrm{w} / \mathrm{v})$ SDS) and placed on top of $12.5 \%(\mathrm{w} / \mathrm{v})$ resolving gels and set using a $1 \%(\mathrm{w} / \mathrm{v})$ agarose sealing gel. The gel electrophoretic separation of the skeletal muscle proteome in the second dimension was carried out by standard SDS-PAGE using an Amersham Ettan DALT-Twelve system (Doran et al., 2006); 12\% slab gels were electrophoresed for $2 \mathrm{~h}$ at $30 \mathrm{~V}$, followed by an $80 \mathrm{~V}$ step until the bromophenol blue dye front had just ran off the gel. Colloidal Coomassie staining of protein gels was carried out according to Neuhoff et al. (1988), and densitometric scanning was performed with a Typhoon Trio variable mode imager from Amersham Biosciences/GE Healthcare. Cropping of gel images was carried out with ImageQuant TL software.

\section{Lectin blotting and immunoblot analysis}

Muscle proteins separated by two-dimensional gel electrophoresis were transferred to Immobilon NC-pure nitrocellulose membranes using a Transblot Cell from Biorad Laboratories (Hemel Hempstead, Herts., UK) at $100 \mathrm{~V}$ for $70 \mathrm{~min}$ (Towbin et al., 1979). To evaluate transfer efficiency, membrane sheets were reversibly stained with $0.1 \%$ (w/v) Ponceau-S Red solution. Removal of dye was achieved by several washes in phosphate-buffered saline (PBS; $0.9 \%(\mathrm{w} / \mathrm{v}) \mathrm{NaCl}$, $50 \mathrm{mM}$ sodium phosphate, $\mathrm{pH}$ 7.4). Blots were then used for lectin labeling or immunoblot analysis. For lectin blotting, nitrocellulose sheets were blocked overnight with $0.5 \%(\mathrm{v} / \mathrm{v})$ Tween 20 in PBS. Incubation with peroxidase-conjugated WGA and UEA-1, was carried out for $3 \mathrm{~h}$ at room temperature (Gravel et al., 1994). Lectin stock solutions containing $1 \mathrm{mg} / \mathrm{ml}$ protein were diluted 1:500 and 1:1000 for UEA-1 and WGA staining, respectively. Blots were washed 6 times for $10 \mathrm{~min}$ in $0.1 \%(\mathrm{v} / \mathrm{v})$ Tween in PBS. Specificity of lectin staining was determined by control experiments in the presence of $1 \mathrm{M} \mathrm{N}$-acetylglucosamine in the case of WGA and $1 \mathrm{M}$ fucose for UEA-1 labeling. Lectin-stained glycans were visualized with the enhanced chemiluminescence technique using the Pierce Supersignal ECL kit as per the manufacturer's instructions. For immunoblottting, nitrocellulose sheets were blocked for $2 \mathrm{~h}$ in $5 \%(\mathrm{w} / \mathrm{v})$ fat-free milk powder dissolved in PBS and then incubated for $3 \mathrm{~h}$ in sufficiently diluted primary antibodies to pyruvate kinase, followed by two 10-min washes in blocking solution (Dowling et al., 2004). Subsequently, blots were incubated with peroxidaseconjugated secondary antibodies for $1 \mathrm{~h}$, washed twice for $10 \mathrm{~min}$ in blocking solution, washed again twice for $10 \mathrm{~min}$ in PBS, and then immuno-decoration was visualized by enhanced chemiluminescence.

\section{Mass spectrometric peptide fingerprinting analysis}

Skeletal muscle glycoproteins, that had exhibited an interaction with peroxidase-conjugated WGA during lectin blotting, were identified by mass spectrometric peptide fingerprinting analysis (Webster and Oxley, 2005) using an Ettan MALDI-ToF Pro instrument from Amersham Biosciences. For proteomic analysis, gel plugs containing a protein spot of interest were placed in $1.5-\mathrm{ml}$ pre-siliconized plastic tubes and then desalted, destained, washed, dried, digested and mixed with matrix by an optimized procedure previously described in detail by our laboratory (Donoghue et al., 2005; Doran et al., 2004, 2006). Glycoproteins were digested with $1 \mu \mathrm{g}$ trypsin per $20 \mu \mathrm{l}$ resuspension buffer and $300 \mu \mathrm{l} 50 \mathrm{mM} \mathrm{NH} \mathrm{HCO}_{3}$ for $1 \mathrm{~h}$ at $37^{\circ} \mathrm{C}$, and then incubated overnight at $37^{\circ} \mathrm{C}$ with $15 \mu \mathrm{l} \quad 50 \mathrm{mM}$ $\mathrm{NH}_{4} \mathrm{HCO}_{3}$ after the removal of excess trypsin solution. The resulting peptide mixture was eluted onto the sample plate with an equal volume of matrix solution containing $5 \mathrm{mg} / \mathrm{ml} \alpha$-cyano-4-hydroxycinnamic acid in $50 \%(\mathrm{v} / \mathrm{v})$ acetonitrile $/ 0.1 \%(\mathrm{v} / \mathrm{v})$ trifluoroacetic acid. The positive reflector mode was used for the recording of mass spectra with an accelerating voltage of $20 \mathrm{kV}$ and pulsed extraction on (focus mass of 2500). For convenient internal calibration, peptide products from trypsin autolysis activity at 221.104 and $842.50 \mathrm{~m} / z$ were used (Doran et al., 2008). External peptides were also employed for calibration purposes. Peptidemix-1 from Laser biolabs contains suitable peaks ranging from 1046.5 to $2465.10 \mathrm{~m} / \mathrm{z}$. Analysis of the mass spectra was performed with Ettan MALDI evaluation software. For the identification of individual protein species, the ProFound database search engine for peptide mass fingerprints was employed.

\section{Confocal microscopy}

Young adult and aged skeletal muscle samples were mounted on cyrocassettes with OCT compound, and transverse cryosections of $10 \mu \mathrm{m}$ thickness were prepared with the help of a Shandon Cryotome (Life Sciences International, Cheshire, UK) at $-20^{\circ} \mathrm{C}$. Muscle sections were then mounted on Superfrost Plus positively charged slides and immediately used for immunoflorescence labeling experiments. Fixation was 
achieved by incubation in ice-cold acetone for $5 \mathrm{~min}$. Tissue sections were left to dry at room temperature before being washed briefly by being submerged in icecold PBS. After the removal of excess PBS, a layer of wax was drawn around the sample with a Liquid Blocker Super PAP Pen. Incubation for $30 \mathrm{~min}$ with 1:50 diluted fluorescein-conjugated WGA (1 mg protein $/ \mathrm{ml}$ ) in the presence or absence of $0.8 \mathrm{M} \mathrm{N}$-acetylglucosamine was performed by an established method (Ohlendieck and Campbell, 1991). For immuno-decoration, sections were first blocked in $0.2 \%(\mathrm{w} / \mathrm{v})$ bovine serum albumin (BSA), $0.2 \%(\mathrm{v} / \mathrm{v})$ Triton $\mathrm{X}-100$, and $2.5 \%(\mathrm{v} / \mathrm{v})$ goat serum in PBS for $30 \mathrm{~min}$, and subsequently incubated with appropriately diluted antibodies to pyruvate kinase or $\beta$-dystroglycan for $4 \mathrm{~h}$, carefully washed twice for $30 \mathrm{~min}$ in $0.2 \%(\mathrm{w} / \mathrm{v}) \mathrm{BSA}$ and $0.2 \%(\mathrm{v} / \mathrm{v})$ Triton X-100 in PBS, incubated for $1 \mathrm{~h}$ with Alexa Fluor dyeconjugated secondary antibodies and then washed again for $30 \mathrm{~min}$ prior to microscopy (Doran et al., 2008). To label nuclei, sections were incubated with $1 \mu \mathrm{g} / \mathrm{ml}$ DAPI for $30 \mathrm{~min}$, washed briefly with PBS, and finally covered by one drop PPD glycerol and a coverslip. Image acquisition and fluorescent scanning was carried out with an Olympus FluoView FV1000 confocal laser scanning microscope (Olympus Life and Material Science Europe, Hamburg, Germany) using the Olympus FluoView Version 1.3c software package.

\section{Results}

\section{Lectin-based 2-D gel electrophoretic analysis of the aged muscle glycoproteome}

$\mathrm{N}$-Glycosylation represents one of the most important post-translational modifications in mammalian cells. Here, we investigated potential alterations in the glycoproteome from aged skeletal muscle. Since lectins interact with complex carbohydrates in a highly specific manner, peroxidase-conjugated WGA was employed to selectively determine altered levels of sialic acid and $\mathrm{N}$-acetylglucosaminyl sugar residues. Fig. 1 shows the gel electrophoretically separated soluble skeletal muscle proteome, which has been stained comparatively with colloidal Coomassie Blue versus WGA. During the initial optimization of lectin labeling of two-dimensional gels, it was revealed that muscle proteins in the low pI range do not exhibit WGA labeling of sufficient intensity for a detailed lectin staining approach (not shown). Therefore, the analysis was restricted to a $\mathrm{pH}$ gradient of $6-11$, which is illustrated in Fig. 1. Although Coomassie staining showed comparable expression levels of major muscle proteins in young adult (Fig. 1A) versus senescent (Fig. 1B) fibers, small changes in certain minor protein species can be observed and have previously been extensively documented (Piec et al., 2005; Doran et al., 2008). The subsequent incubation of similar gels with peroxidase-conjugated WGA resulted in a reproducible labeling pattern of distinct 2-D protein spots in young adult (Fig. 1C) versus senescent (Fig. 1D) skeletal muscle. Major muscle proteins, which had been recognized by the lectin, were then identified by peptide mass fingerprinting analysis.

\section{Mass spectrometric identification of major components of the muscle glycoproteome}

The Coomassie-stained master gel of Fig. 1A shows thirteen 2-D protein spots, which had been recognized by WGA and could unequivocally be identified by MALDI-ToF mass spectrometric analysis using in-gel tryptic digests. The spot numbering in Fig. 1 relates to the information given in Table 1 on individual 2-D spots with respect to protein identification, sequence coverage, apparent molecular mass, predicted $\mathrm{pI}$, and the statistical significance of the peptide mass fingerprinting analysis, as well as the international protein accession number. The identified muscle components belong mostly to the family of metabolic enzymes. This included glycolytic enzymes, such as pyruvate kinase (spots 2 and 3), enolase (spots 4 and 5), phosphoglycerate kinase (spot 7), aldolase (spot 8), glyceraldehyde-3phosphate dehydrogenase (spots 9 and 10), and phosphoglycerate mutase (spot 12). A key enzyme of the citric acid cycle, aconitase (spot 1), the essential muscle protein involved in carbon dioxide removal, carbonic anhydrase (spot 13), and an essential component of nucleotide metabolism, creatine kinase (spot 6), were also shown to be labeled by the WGA probe.

\section{Proteomic analysis of pyruvate kinase isozyme M1 in aged skeletal muscle}

The densitometric analysis of the 2-D protein expression profile of young adult versus senescent muscle revealed that only one fiber element, i.e., spot 2 (pI 6.6/ $57.8 \mathrm{kDa}$ ), out of the total cohort of WGA-labeled proteins exhibited a drastic change in glycosylation levels. This protein was identified by mass spectrometric peptide fingerprinting as one of the key rate-limiting enzymes of glycolysis, the M1 isoform of pyruvate kinase (gi|16757994|). Fig. 2A shows the mass spectrometric spectrum of the M1 isozyme and lists the masses of the tryptic fragments that correlate with the amino acid sequence of this muscle enzyme. The coverage of the entire sequence by identified peptides of this large protein was approximately $21 \%$ (Fig. 2B), which lends credence to a positive identification. 

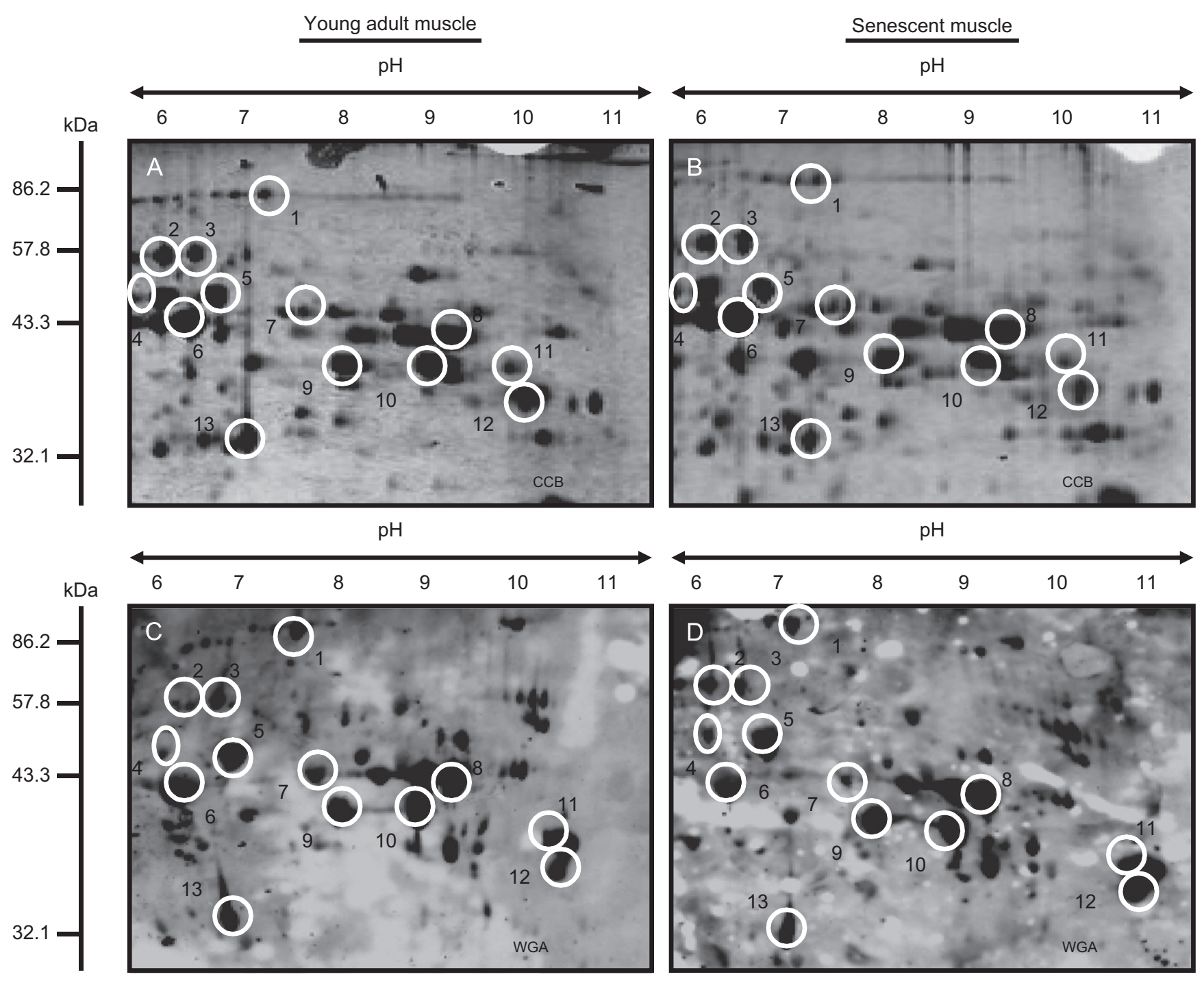

Fig. 1. Lectin-based proteomic profiling of young adult versus aged skeletal muscle. Two-dimensional gels of young adult (A, C) versus senescent (B, D) gastrocnemius rat muscle, stained with colloidal Coomassie Blue (CCB; A, B) or peroxidase-conjugated WGA (C, D). Major lectin-labeled protein species, which could be identified by proteomic methodology, are marked by circles and numbered 1-13. The mass spectrometric identification of these muscle glycoproteins is listed in Table 1 . The $\mathrm{pH}$ values of the first dimension gel system and molecular mass standards (in $\mathrm{kDa}$ ) of the second dimension are indicated on the top and on the left of the panels, respectively.

\section{Comparative analysis of pyruvate kinase isozyme M1 in young adult versus aged muscle}

Following the proteomic identification of muscle pyruvate kinase as a protein with a drastically altered level of $\mathrm{N}$-glycosylation in aged fibers, protein expression of this enzyme was compared to lectin labeling intensity to quantitate this finding. Fig. 3 shows representative lectin- and immuno-decoration analyses, and Fig. 4 graphically represents the statistical evaluation of these blotting experiments. In analogy to the antibody labeling of this glycolytic enzyme (Fig. 3I and J), lectin blots illustrated that both WGA (Fig. 3A and B) and UEA-1 (Fig. 3E and F) recognize three main pyruvate kinase species. Specificity of lectin staining was shown by incubation of control blots with the competitive sugars $\mathrm{N}$-acetylglucosamine (Fig. 3C and D) and fucose (Fig. $3 \mathrm{G}$ and $\mathrm{H}$ ), which abolished the interactions of UEA-1 and WGA, respectively. Antibodies to the M1 isozyme of pyruvate kinase usually decorate five distinct protein spots of similar molecular mass but differing $\mathrm{pI}$ (not shown), whereby the three immuno-labeled protein species in Fig. 3I and $\mathbf{J}$ (pI 6.0, 6.6 and 7.2) represent the major forms. An attempt to determine the change in molecular mass of pyruvate kinase isozyme M1 due to $\mathrm{N}$-glycosylation using de-glycosylation assays failed (not shown). The two minor pyruvate kinase-containing 2-D spots with a lower pI were not co-stained by lectins, 
Table 1. Glycoproteins labeled by peroxidase-conjugated WGA in both 3-month- and 30-month-old rat skeletal muscle

\begin{tabular}{|c|c|c|c|c|c|c|}
\hline $\begin{array}{l}\text { Spot } \\
\text { no. }\end{array}$ & Name of identified protein & $\begin{array}{l}\text { Sequence } \\
\text { coverage }(\%)\end{array}$ & $\begin{array}{l}\text { Molecular } \\
\text { mass }(\mathrm{kDa})\end{array}$ & $\mathrm{p} I$ & $\begin{array}{l}\text { Expectation } \\
\text { value }\end{array}$ & $\begin{array}{l}\text { Protein } \\
\text { accession no. }\end{array}$ \\
\hline 1 & Aconitase 2, mitochondrial & 22.3 & 86.16 & 8.2 & $1 e-004$ & gi $|38541404|$ \\
\hline 2 & Pyruvate kinase & 21.0 & 57.8 & 6.6 & $6.2 \mathrm{e}-003$ & gi| $16757994 \mid$ \\
\hline 3 & Pyruvate kinase & 30.0 & 57.8 & 7.2 & $1.9 \mathrm{e}-004$ & gi $|206205|$ \\
\hline 4 & Enolase 3 & 27.0 & 47.0 & 7.8 & $1.5 \mathrm{e}-003$ & gi|6978811| \\
\hline 5 & Enolase 3 & 30.0 & 47.0 & 7.1 & $4.4 \mathrm{e}-003$ & gi $|54035288|$ \\
\hline 6 & Creatine kinase & 25.0 & 43.3 & 6.6 & $6.7 e-003$ & gi $|6978661|$ \\
\hline 7 & Phosphoglycerate kinase 1 & 36.5 & 44.9 & 8.3 & 0.001 & gi $|38649310|$ \\
\hline 8 & Aldolase A & 27.0 & 39.7 & 8.8 & $5.3 e-003$ & gi $|202837|$ \\
\hline 9 & $\begin{array}{l}\text { Glyceraldehyde-3-phosphate } \\
\text { dehydrogenase }\end{array}$ & 27.3 & 36.1 & 8.7 & 0.009 & gi $|56188|$ \\
\hline 10 & $\begin{array}{l}\text { Glyceraldehyde-3-phosphate } \\
\text { dehydrogenase }\end{array}$ & 18.9 & 36.1 & 8.7 & 0.019 & gi $|62653546|$ \\
\hline 11 & Similar to mKIAA0613 protein & 36.4 & 30.9 & 9.2 & $1 e-004$ & gi $|54035563|$ \\
\hline 12 & Phosphoglycerate mutase 2 & 41.0 & 28.9 & 9.0 & $1.2 \mathrm{e}-003$ & gi $|893948|$ \\
\hline 13 & Carbonic anhydrase & 55.4 & 29.4 & 6.9 & $1 \mathrm{e}-004$ & gi|31377484| \\
\hline
\end{tabular}

A

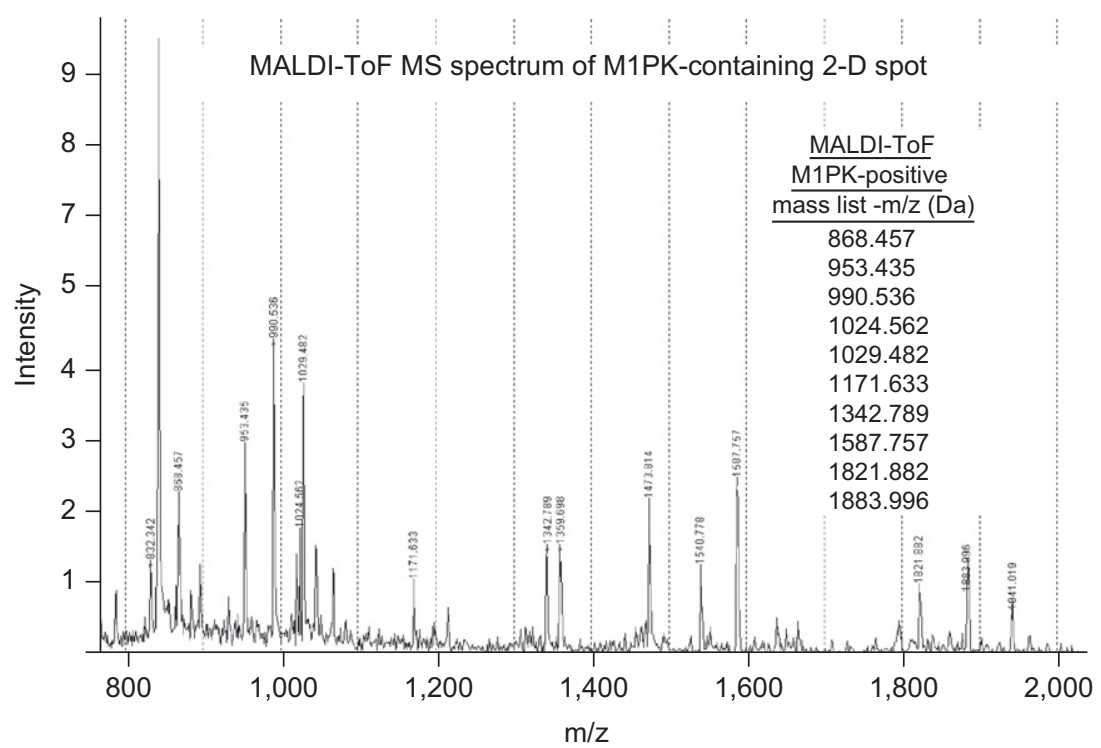

B Peptide mass fingerprint of M1PK from in-gel digest

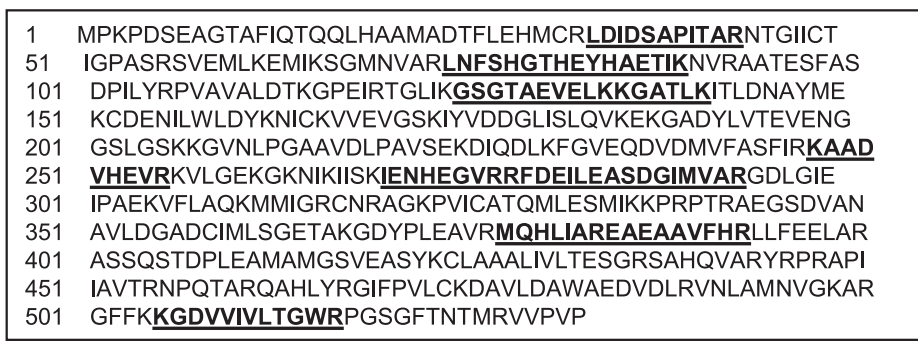

Amino acid sequence of gi|16757994| Pyruvate kinase, skeletal muscle

M1 isoform; Rattus sp.; pl 6.6; $57.8 \mathrm{kDa}$

Fig. 2. MALDI-ToF mass spectrometric identification of pyruvate kinase isozyme M1 from skeletal muscle.

probably due to their low abundance. The existence of muscle pyruvate kinase isoforms with different $\mathrm{pI}$ but comparable molecular masses suggests the presence of varying degrees of charge-inducing post-translational modifications, such as phosphorylation. This agrees with findings of a recent phosphoproteomic analysis of 
aged skeletal muscle fibers employing the phosphospecific Pro-Q Diamond dye. This study has identified muscle pyruvate kinase as one of 59 highly phosphory-

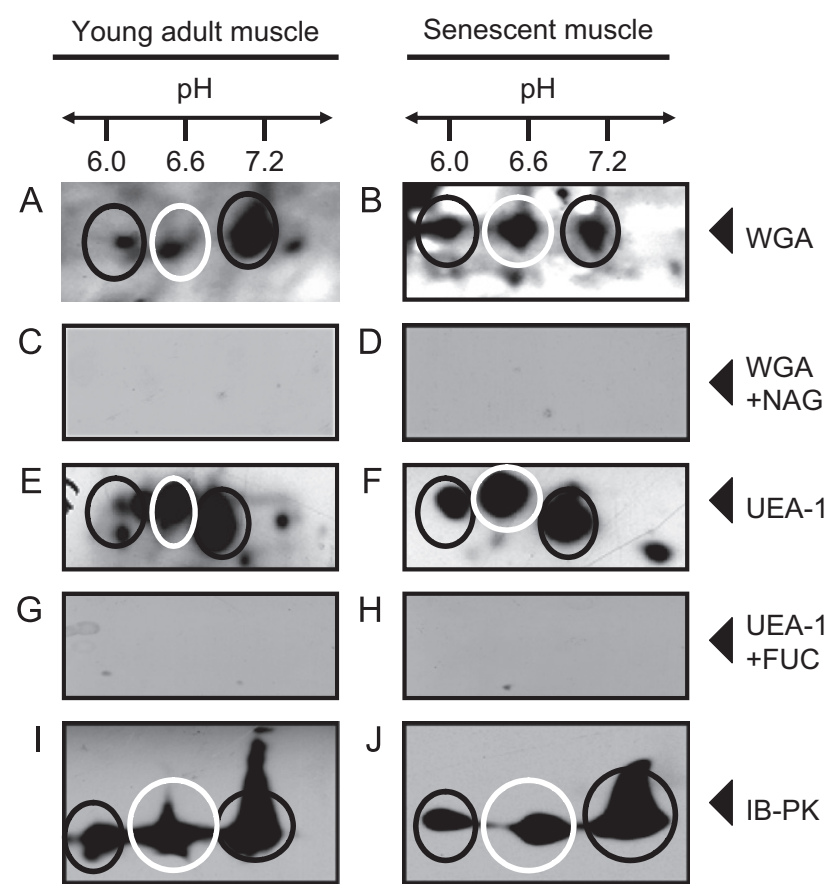

Fig. 3. Comparative two-dimensional lectin blotting and immunoblot analysis of pyruvate kinase in aged skeletal muscle. Expanded view of two-dimensional blots of young adult (A, C, $\mathrm{E}, \mathrm{G}, \mathrm{I})$ versus senescent $(\mathrm{B}, \mathrm{D}, \mathrm{F}, \mathrm{H}, \mathrm{J})$ skeletal muscle preparations, labeled with peroxidase-conjugated WGA (A-D), peroxidase-conjugated UEA-1 (E-H), or an antibody to pyruvate kinase (PK; I, J). Control experiments in the presence of the competitive sugars $N$-acetylglucosamine (NAG) or fucose (FUC) are shown in $(\mathrm{C}, \mathrm{D})$ and $(\mathrm{G}, \mathrm{H})$, respectively. The lectinor antibody-labeled M1 isoform of pyruvate kinase with a pI of 6.6 is marked by white circles and the two other identified pyruvate kinase isoforms by black circles. lated protein species in total extracts from contractile fibers (Gannon et al., 2008). In contrast to relatively comparable UEA-1 staining in young adult versus aged preparations (Fig. 4), protein spots representing pyruvate kinase showed a marked increase in WGA labeling with the most drastic elevation of $\mathrm{N}$-glycosylation in the M1 species with a $\mathrm{pI}$ of 6.6. In contrast to increased WGA staining of the M1 isozyme, immunoblot analysis revealed a decreased protein expression level for this highly glycosylated pyruvate kinase isoform in aged muscle (Fig. 4).

\section{Decreased protein concentration and enzyme activity of pyruvate kinase in aged muscle}

Confocal microscopy and enzymatic testing was used to investigate the subcellular localization and activity of pyruvate kinase in young adult versus senescent muscle. Fig. 5 demonstrates an age-related reduction in pyruvate kinase activity. The key ATP-producing step in muscle glycolysis is clearly diminished in 30-month- versus 3-month-old gastrocnemius preparations (Fig. 5A and B). The specificity of the coupled enzyme assay system employed in this study is shown by the lack of activity in control experiments (Fig. 5C). The graphical presentation in Fig. 5D shows an approximately 50\% reduction in pyruvate kinase activity in aged fibers. Although enzymatic studies with dilute solutions of isolated muscle fractions do not take into account the potential influence of molecular crowding or cooperative kinetics (Ellis, 2001; Despa et al., 2005) on pyruvate kinase activity in its cellular context, this result nevertheless represents a strong indication that the changed protein expression level and/or altered post-translational modifications have an effect on the glycolytic pathway. Confocal microscopy was employed for investigating lectin labeling of
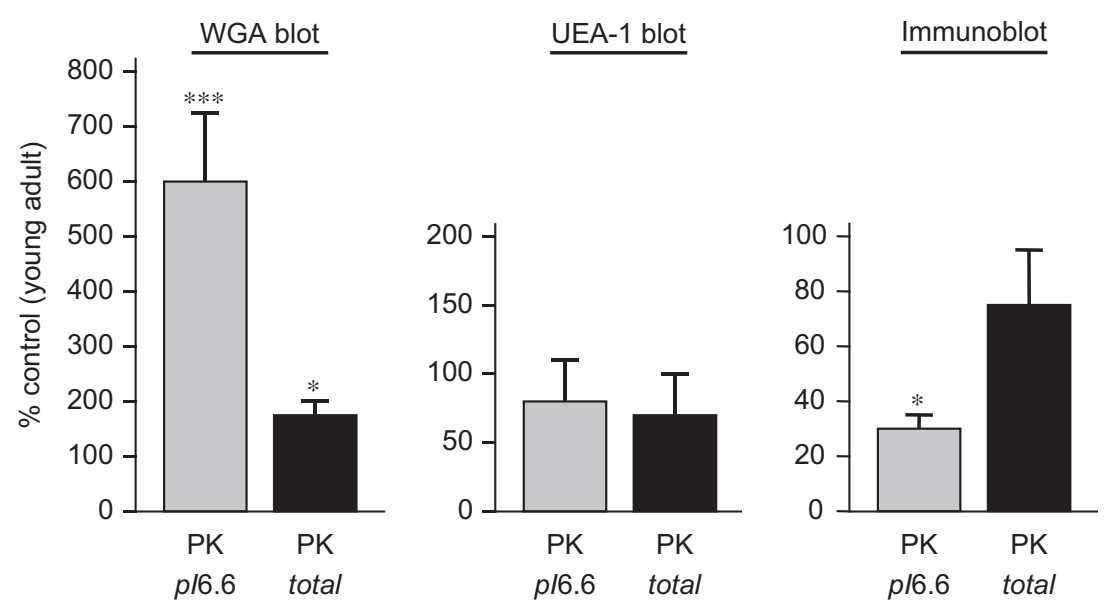

Fig. 4. Quantification and statistical evaluation of lectin and immunoblot analysis of pyruvate kinase M1 isozyme in senescent gastrocnemius muscle preparations, as compared to control young adult samples. Both, labeling of the M1 isozyme and total staining of all three main pyruvate kinase isoforms is shown. The comparative blotting was statistically evaluated using an unpaired Student's $t$-test $(n=6 ; * p<0.05 ; * * * p<0.001)$. 
A

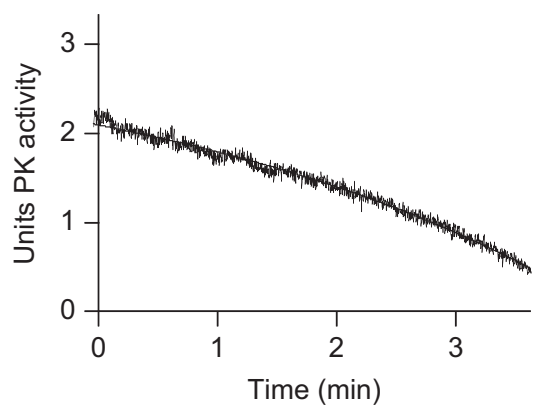

C

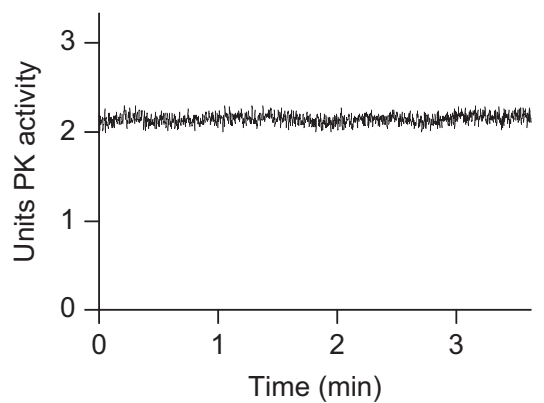

B

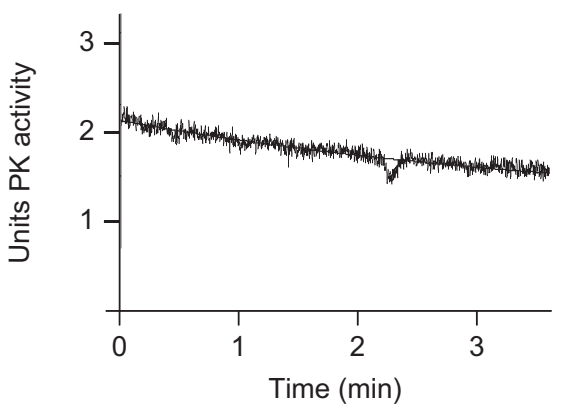

D

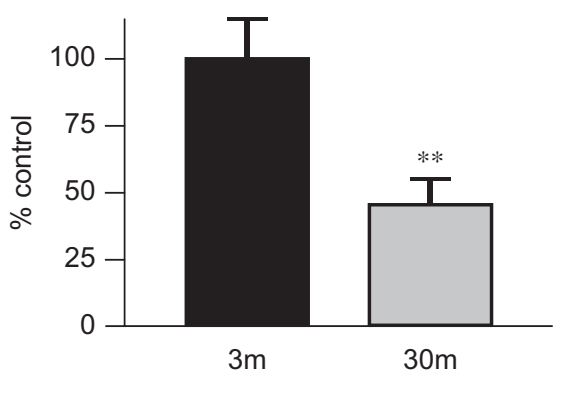

Fig. 5. Comparison of pyruvate kinase activity in young adult (A) versus senescent (B) gastrocnemius muscle preparations. Enzyme activity was determined with a coupled lactate dehydrogenase assay system, whereby the decrease in NADH levels corresponds to the production rate of pyruvate via pyruvate kinase activity. (C) Control experiment without muscle protein present in the assay suspension. (D) Statistical evaluation of the comparative enzyme testing using an unpaired Student's $t$-test $\left(n=6\right.$; $\left.{ }^{* *} p<0.005\right)$.

muscle fibers and the subcellular localization of pyruvate kinase using primary antibodies to the M1 isozyme. Fluorescein-conjugated WGA staining was comparable between young adult and senescent muscle and labeled almost exclusively the cellular periphery (Fig. 6A and C), as previously shown (Ohlendieck and Campbell, 1991). Incubation of muscle cryosections with WGA in the presence of its competitive sugar, $N$-acetylglucoseamine, did not result in any robust labeling patterns (Fig. 6B and D), showing the specificity of lectin staining. The fiber periphery and nuclei of both young adult and senescent gastrocnemius muscle were visualized by immunolabeling of the sarcolemmal marker $\beta$-dystroglycan and DAPI staining, respectively (Fig. 6E and F). Comparative immunofluorescence labeling of pyruvate kinase revealed decreased levels in aged fibers (Fig. 6E and F). Fluorescence scanning suggests an approximate $40 \%$ reduction of this glycolytic enzyme in aged skeletal muscle (Fig. 6G). The decreased levels of pyruvate kinase isozyme M1 in senescent fibers agree with the above outlined gel electrophoretic and immunoblot analysis.

\section{Discussion}

The multi-factorial muscle wasting pathology observed in aged fibers is extremely complex and is affected by a great variety of molecular and cellular changes
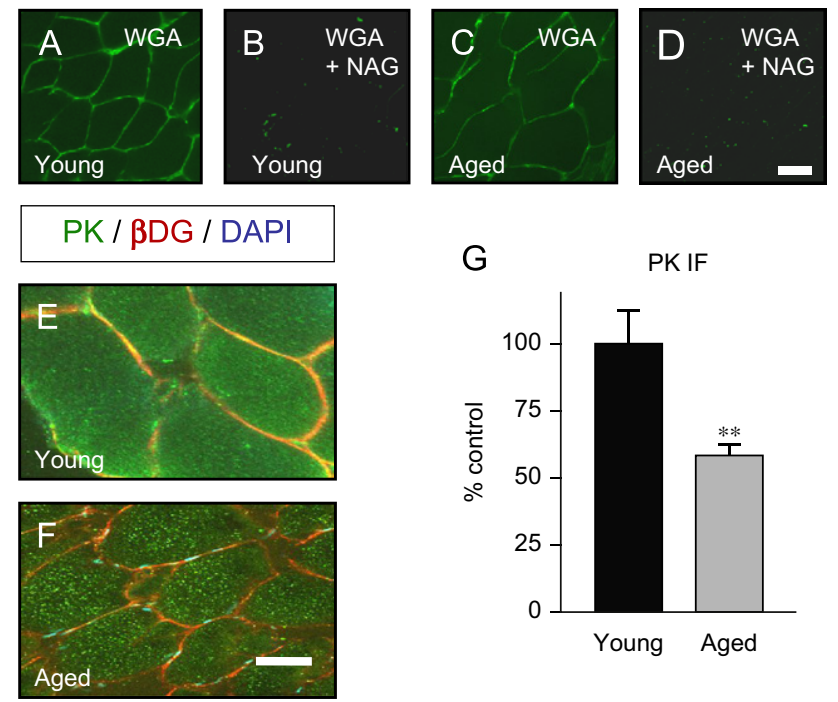

Fig. 6. Indirect immunofluorescence localization of WGAlabeled proteins $(\mathrm{A}-\mathrm{D})$, the surface membrane marker $\beta$ dystroglycan $(\beta$-DG; E, F) and pyruvate kinase isoyzme M1 $(\mathrm{PK} ; \mathrm{E}, \mathrm{F})$ in young adult $(\mathrm{A}, \mathrm{B}, \mathrm{E})$ versus senescent $(\mathrm{C}, \mathrm{D}, \mathrm{F})$ gastrocnemius muscle. Lectin labeling was performed in the absence (A, C) or presence (B, D) of the competitive sugar $0.8 \mathrm{M} \mathrm{N}$-acetylglucosamine. DAPI staining of nuclei is shown in the merged images (E, F). Bars: $40 \mu \mathrm{m}$. (G) Fluorescence intensity for pyruvate kinase labeling. The statistical evaluation was carried out with an unpaired Student's $t$-test $(n=6$; $* * p<0.005)$. 
(Carmeli et al., 2002; Edstrom et al., 2007). This includes increased oxidative stress, mitochondrial abnormalities, disturbed microcirculation, hormonal imbalance, incomplete ion homeostasis, denervation, and impaired excitation-contraction coupling (Larsson, 1998; Degens, 1998; Squier and Bigelow, 2000; Bua et al., 2002; Delbono, 2002), as well as a decreased regenerative potential (Renault et al., 2002; Lorenzon et al., 2004; Beccafico et al., 2007). In addition, altered posttranslational modifications, such as tyrosine nitration, were recently described as occuring in an age-related manner on numerous skeletal muscle proteins (Kanski et al., 2003, 2005). In analogy, we have investigated here potential changes in another frequent post-translational modification, i.e., $\mathrm{N}$-glycosylation, by analyzing the glycoproteome of aged muscle fibers. Since glycosylation plays a central role in normal and pathological processes in skeletal muscle, it is plausible that altered glycoprotein expression levels may also contribute to the molecular pathogenesis of sarcopenia.

The modification of key muscle proteins by oligosaccharide attachment has an effect on their secondary and tertiary folding patterns, the formation of quaternary structures and supramolecular assemblies. This in turn influences the overall stability of protein structure, enzymatic regulation and activity, and the subcellular targeting of glycoproteins (Ohtsubo and Marth, 2006). The lectin-based proteomic profiling of aged skeletal muscle outlined in this report has revealed that, although the pyruvate kinase protein species with a pI of 6.6 is less abundant in aged fibers, this isozyme exhibits drastically increased levels of $N$-glycosylation. During the initial optimization phase of this project, intense and reproducible WGA labeling was mostly achieved with skeletal muscle proteins with a $\mathrm{pI}$ greater than 7. Muscle proteins with a lower $\mathrm{pI}$ were only poorly recognized by the lectin labeling procedure. This could possibly be due to a reduced binding affinity of peroxidase-conjugated lectins to more acidic proteins. Alternatively, acidic muscle proteins might exhibit less $N$-glycosylation as compared to components with a more basic pI. This issue will be addressed by refined lectin-protein binding studies in the future. Here, we have focused on proteins that can be properly labeled with WGA in the $\mathrm{pH}$ range of 6 to 11 . The differential labeling of the aged M1 isoform by UEA-1 versus WGA suggests that fucose-containing carbohydrate moieties are relatively comparable between young adult and aged muscle, but increased levels of sialic acid and $\mathrm{N}$-acetylglucosaminyl sugar residues are associated with fiber aging.

Mammalian pyruvate kinase is a ubiquitous enzyme with ATP-pyruvate 2-O-phosphotransferase activity (EC 2.7.1.40) that exists as four separate isozymes (Munoz and Ponce, 2003). In skeletal muscle, the M1 isoform is a major constituent of the soluble fraction of the fiber proteome (Maughan et al., 2005). During the final step of glycolysis, its enzymatic reaction yields pyruvate and ATP from phosphoenolpyruvate, ADP and a proton (Erlandsen et al., 2000). The crystal structure of the muscle isoform has been determined from cat at a resolution of $2.6 \AA$ (Stuart et al., 1979) and rabbit at a resolution of $2.1 \AA$ (Larsen et al., 1998). The functional enzyme is represented by a homotetrameric assembly, whereby the M1 isoform consists of 530-residue subunits that can be further divided into a four-domain structure, i.e., N-domain (aa 1-42), A-domain (aa 43-115, aa 224-387), B-domain (aa 116-223) and C-domain (aa 388-530). The active site of the enzyme is formed within a cleft between the two large domains A and B (Larsen et al., 1998). With respect to sustainable muscle performance, the central metabolic position of pyruvate kinase is crucial for both glycolysis and oxidative phosphorylation (Conley et al., 2001). The bioenergetic provision of ATP for muscular activity is secured in three main ways: (i) anaerobic glycolysis, (ii) creatine kinase activity, and (iii) oxidative phosphorylation. Thus, glycolysis is not only essential for the initial steps of anaerobic glucose metabolism, but also as a feeder pathway for subsequent mitochondriabased ATP production. A study by Lanza et al. (2005) has shown that the glycolytic flux rate and ATP production from anaerobic glycolysis is drastically reduced in aged muscle fibers. This agrees with the findings presented here. Possibly, the reduced expression of the M1 pyruvate kinase and its altered posttranslational modifications, i.e., $\mathrm{N}$-glycosylation and tyrosine nitration (Kanski et al., 2005), might play a role in this metabolic shift during muscle aging.

The recent fluorescence difference in-gel electrophoretic analysis of young adult versus aged muscle has clearly revealed decreased levels of key glycolytic enzymes and the concomitant up-regulation of citric acid cycle components and elements of oxidative phosphorylation (Doran et al., 2008), suggesting a transition to more aerobic-oxidative metabolism in a slower-twitching senescent fiber population. The drastic increase in the oxygen transporter myoglobin and the fatty acid-binding protein also agrees with this idea (Doran et al., 2008), and was previously established as a reliable indicator of fiber type transformation (Donoghue et al., 2005, 2007). Reduced expression levels of the pyruvate kinase M1 isozyme and its altered glycosylation pattern, as revealed in this report by a combination of colloidal Coomassie and WGA lectin labeling, supports the hypothesis that an altered glycolytic flux rate is associated with sarcopenia of old age. However, since many mammalian proteins are multi-functional, altered pyruvate kinase expression might also influence other physiological processes. For example, the monomeric form of this enzyme has been shown to also function as a binding protein for thyroid 
hormone (Parkison et al., 1991). Thus, the regulation of the transcriptional response of the thyroid hormone nuclear receptor (Ashizawa et al., 1992) may also be altered in aged tissue.

In conclusion, skeletal muscle aging is associated with drastic changes in the protein expression level and $\mathrm{N}$-glycosylation of a major glycolytic enzyme, the M1 isozyme of pyruvate kinase. The decreased protein concentration and elevated glycosylation of this ratelimiting enzyme might be involved in the metabolic shift of aged fibers. Increased glycosylation may influence the proper subcellular targeting of the enzyme, its functionally important intra- and inter-molecular interactions, overall enzyme stability, and efficient coupling between substrates and active site, thereby negatively affecting glycolytic activity. The results presented in this study agree with the previously observed reduced glycolytic flux rate in senescent muscle and an age-related shift to more aerobic-oxidative metabolism.

\section{Acknowledgments}

Research was supported by a principal investigator grant from Science Foundation Ireland (SFI-04/IN3/ B614) and equipment grants from the Irish Health Research Board (HRB-EQ/2003/3, HRB-EQ/2004/2). The authors thank Dr. Marina Lynch (Trinity College Dublin) for her generous help obtaining aged rat muscle and Dr. Ica Dix (NUI Maynooth) for help with confocal microscopy.

\section{References}

Ambrosi, M., Cameron, N.R., Davis, B.G., 2005. Lectins: tools for the molecular understanding of the glycocode. Org. Biomol. Chem. 3, 1593-1608.

Ashizawa, K., Fukuda, T., Cheng, S.Y., 1992. Transcriptional stimulation by thyroid hormone of a cytosolic thyroid hormone binding protein which is homologous to a subunit of pyruvate kinase M1. Biochemistry 31, 2774-2778.

Bains, G., Lee, R.T., Lee, Y.C., Freire, E., 1992. Microcalorimetric study of wheat germ agglutinin binding to $N$ acetylglucosamine and its oligomers. Biochemistry 31 , 12624-12628.

Barresi, R., Michele, D.E., Kanagawa, M., Harper, H.A., Dovico, S.A., Satz, J.S., Moore, S.A., Zhang, W., Schachter, H., Dumanski, J.P., Cohn, R.D., Nishino, I., Campbell, K.P., 2004. LARGE can functionally bypass alpha-dystroglycan glycosylation defects in distinct congenital muscular dystrophies. Nat. Med. 10, 696-703.

Beccafico, S., Puglielli, C., Pietrangelo, T., Bellomo, R., Fano, G., Fulle, S., 2007. Age-dependent effects on functional aspects in human satellite cells. Ann. NY Acad. Sci. 1100, 345-352.

Bergmeyer, H.U., Bernt, E., 1974. Pyruvate kinase. In: Bergmeyer, H.U. (Ed.), Methods of Enzymatic Analysis, vol. 1. Academic Press, New York, pp. 509-510.
Bhavanandan, V.P., Katlic, A.W., 1979. The interaction of wheat germ agglutinin with sialoglycoproteins. The role of sialic acid. J. Biol. Chem. 254, 4000-4008.

Bond, M.R., Kohler, J.J., 2007. Chemical methods for glycoprotein discovery. Curr. Opin. Chem. Biol. 11, 52-58.

Brockington, M., Muntoni, F., 2005. The modulation of skeletal muscle glycosylation as a potential therapeutic intervention in muscular dystrophies. Acta Myol. 24, 217-221.

Bua, E.A., McKiernan, S.H., Wanagat, J., McKenzie, D., Aiken, J.M., 2002. Mitochondrial abnormalities are more frequent in muscles undergoing sarcopenia. J. Appl. Physiol. 92, 2617-2624.

Budnik, B.A., Lee, R.S., Steen, J.A., 2006. Global methods for protein glycosylation analysis by mass spectrometry. Biochim. Biophys. Acta 1764, 1870-1880.

Carmeli, E., Coleman, R., Reznick, A.Z., 2002. The biochemistry of aging muscle. Exp. Gerontol. 37, 477-489.

Conley, K.E., Kemper, W.F., Crowther, G.J., 2001. Limits to sustainable muscle performance: interaction between glycolysis and oxidative phosphorylation. J. Exp. Biol. 204, 3189-3194.

Cummings, R.D., 1994. Use of lectins in analysis of glycoconjugates. Methods Enzymol. 230, 66-86.

Degens, H., 1998. Age-related changes in the microcirculation of skeletal muscle. Adv. Exp. Med. Biol. 454, 343-348.

Delbono, O., 2002. Molecular mechanisms and therapeutics of the deficit in specific force in ageing skeletal muscle. Biogerontology 3, 265-270.

Deschenes, M.R., 2004. Effects of aging on muscle fibre type and size. Sports Med. 34, 809-824.

Despa, F., Orgill, D.P., Lee, R.C., 2005. Molecular crowding effects on protein stability. Ann. NY Acad. Sci. 1066, 54-66.

Doherty, T.J., 2003. Aging and sarcopenia. J. Appl. Physiol. 95, 1717-1727.

Donoghue, P., Doran, P., Dowling, P., Ohlendieck, K., 2005. Differential expression of the fast skeletal muscle proteome following chronic low-frequency stimulation. Biochim. Biophys. Acta 1752, 166-176.

Donoghue, P., Doran, P., Wynne, K., Pedersen, K., Dunn, M.J., Ohlendieck, K., 2007. Proteomic profiling of chronic low-frequency stimulated fast muscle. Proteomics 7, 3417-3430.

Doran, P., Dowling, P., Lohan, J., McDonnell, K., Poetsch, S., Ohlendieck, K., 2004. Subproteomics analysis of $\mathrm{Ca}^{2+}$. binding proteins demonstrates decreased calsequestrin expression in dystrophic mouse skeletal muscle. Eur. J. Biochem. 271, 3943-3952.

Doran, P., Martin, G., Dowling, P., Jockusch, H., Ohlendieck, K., 2006. Proteome analysis of the dystrophin-deficient MDX diaphragm reveals a drastic increase in the heat shock protein cvHSP. Proteomics 6, 4610-4621.

Doran, P., Gannon, J., O’Connell, K., Ohlendieck, K., 2007a. Proteomic profiling of animal models mimicking skeletal muscle disorders. Proteomics Clin. Appl. 1, 1169-1184.

Doran, P., Gannon, J., O'Connell, K., Ohlendieck, K., 2007 b. Aging skeletal muscle shows a drastic increase in the small heat shock proteins $\alpha \mathrm{B}$-crystallin/HspB5 and cvHsp/ HspB7. Eur. J. Cell Biol. 86, 629-640. 
Doran, P., O’Connell, K., Gannon, J., Kavanagh, M., Ohlendieck, K., 2008. Opposite pathobiochemical fate of pyruvate kinase and adenylate kinase in aged rat skeletal muscle as revealed by proteomic DIGE analysis. Proteomics 8, 364-377.

Dowling, P., Doran, P., Ohlendieck, K., 2004. Drastic reduction of sarcalumenin in Dp427 (dystrophin of $427 \mathrm{kDa}$ )-deficient fibres indicates that abnormal calcium handling plays a key role in muscular dystrophy. Biochem. J. 379, 479-488.

Durham, M., Regnier, F.E., 2006. Targeted glycoproteomics: serial lectin affinity chromatography in the selection of $O$-glycosylation sites on proteins from the human blood proteome. J. Chromatogr. A 1132, 165-173.

Edstrom, E., Altun, M., Bergman, E., Johnson, H., Kullberg, S., Ramirez-Leon, V., Ulfhake, B., 2007. Factors contributing to neuromuscular impairment and sarcopenia during aging. Physiol. Behav. 92, 129-135.

Ellis, R.J., 2001. Macromolecular crowding: obvious but underappreciated. Trends Biochem. Sci. 26, 597-604.

Erlandsen, H., Abola, E.E., Stevens, R.C., 2000. Combining structural genomics and enzymology: completing the picture in metabolic pathways and enzyme active sites. Curr. Opin. Struct. Biol. 10, 719-730.

Freeze, H.H., 2006. Genetic defects in the human glycome. Nat. Rev. Genet. 7, 537-551.

Gagneux, P., Varki, A., 1999. Evolutionary considerations in relating oligosaccharide diversity to biological function. Glycobiology 9, 747-755.

Gannon, J., Staunton, L., O'Connell, K., Doran, P., Ohlendieck, K., 2008. Phosphoproteomic analysis of aged skeletal muscle. Int. J. Mol. Med. 22, 33-42.

Gelfi, C., Vigano, A., Ripamonti, M., Pontoglio, A., Begum, S., Pellegrino, M.A., Grassi, B., Bottinelli, R., Wait, R., Cerretelli, P., 2006. The human muscle proteome in aging. J. Proteome Res. 5, 1344-1353.

Geyer, H., Geyer, R., 2006. Strategies for analysis of glycoprotein glycosylation. Biochim. Biophys. Acta 1764, 1853-1869.

Gravel, P., Walzer, C., Hochstrasser, D.F., Turler, H., Baland, L.P., 1994. Analysis of glycoproteins separated by twodimensional gel electrophoresis using lectin blotting revealed by chemiluminescence. Anal. Biochem. 221, $66-71$.

Greenlund, L.J., Nair, K.S., 2003. Sarcopenia - consequences, mechanisms, and potential therapies. Mech. Ageing Dev. 124, 287-299.

Haliloglu, G., Topaloglu, H., 2004. Glycosylation defects in muscular dystrophies. Curr. Opin. Neurol. 17, 521-527.

Harvey, D.J., 2005. Structural determination of N-linked glycans by matrix-assisted laser desorption/ionization and electrospray ionization mass spectrometry. Proteomics 5, 1774-1786.

Janssen, I., Heymsfield, S.B., Ross, R., 2002. Low relative skeletal muscle mass (sarcopenia) in older persons is associated with functional impairment and physical disability. J. Am. Geriatr. Soc. 50, 889-896.

Kanski, J., Alterman, M.A., Schoneich, C., 2003. Proteomic identification of age-dependent protein nitration in rat skeletal muscle. Free Radical Biol. Med. 35, 1229-1239.
Kanski, J., Hong, S.J., Schoneich, C., 2005. Proteomic analysis of protein nitration in aging skeletal muscle and identification of nitrotyrosine-containing sequences in vivo by nanoelectrospray ionization tandem mass spectrometry. J. Biol. Chem. 280, 24261-24266.

Lanza, I.R., Befroy, D.E., Kent-Braun, J.A., 2005. Age-related changes in ATP-producing pathways in human skeletal muscle in vivo. J. Appl. Physiol. 99, 1736-1744.

Larsson, L., 1998. The age-related motor disability: underlying mechanisms in skeletal muscle at the motor unit, cellular and molecular level. Acta Physiol. Scand. 163, S27-S29.

Larsen, T.M., Benning, M.M., Rayment, I., Reed, G.H., 1998. Structure of the bis $\left(\mathrm{Mg}^{2+}\right)$-ATP-oxalate complex of the rabbit muscle pyruvate kinase at $2.1 \AA$ resolution: ATP binding over a barrel. Biochemistry 37, 6247-6255.

Lorenzon, P., Bandi, E., de Guarrini, F., Pietrangelo, T., Schafer, R., Zweyer, M., Wernig, A., Ruzzier, F., 2004. Ageing affects the differentiation potential of human myoblasts. Exp. Gerontol. 39, 1545-1554.

Martin-Rendon, E., Blake, D.J., 2003. Protein glycosylation in disease: new insights into the congenital muscular dystrophies. Trends Pharmacol. Sci. 24, 178-183.

Maughan, D.W., Henkin, J.A., Vigoreaux, J.O., 2005. Concentrations of glycolytic enzymes and other cytosolic proteins in the diffusible fraction of a vertebrate muscle proteome. Mol. Cell. Proteomics 4, 1541-1549.

Morelle, W., Michalski, J.C., 2007. Analysis of protein glycosylation by mass spectrometry. Nat. Protocols 2, 1585-1602.

Munoz, M.E., Ponce, E., 2003. Pyruvate kinase: current status of regulatory and functional properties. Comp. Biochem. Physiol. B: Biochem. Mol. Biol. 135, 197-218.

Muraki, M., Ishimura, M., Harata, K., 2002. Interactions of wheat-germ agglutinin with GlcNAc beta 1,6 Gal sequence. Biochim. Biophys. Acta 1569, 10-20.

Naeem, A., Saleemuddin, M., Khan, R.H., 2007. Glycoprotein targeting and other applications of lectins in biotechnology. Curr. Protein Pept. Sci. 8, 261-271.

Navarro, A., Lopez-Cepero, J.M., Sanchez del Pino, M.J., 2001. Skeletal muscle and aging. Front. Biosci. 6, D26-D44.

Neuhoff, V., Arold, N., Taube, D., Ehrhardt, W., 1988. Improved staining of proteins in polyacrylamide gels including isoelectric focusing gels with clear background at nanogram sensitivity using Coomassie Brilliant Blue G250 and R-250. Electrophoresis 9, 255-262.

O’Connell, K., Gannon, J., Doran, P., Ohlendieck, K., 2007. Proteomic profiling reveals a severely perturbed protein expression pattern in aged skeletal muscle. Int. J. Mol. Med. 20, 145-153.

Ohlendieck, K., Campbell, K.P., 1991. Dystrophin-associated proteins are greatly reduced in skeletal muscle from MDX mice. J. Cell Biol. 115, 1685-1694.

Ohtsu, I., Nakanisi, T., Furuta, M., Ando, E., Nishimura, O., 2005. Direct matrix-assisted laser desorption/ionization time-of-flight mass spectrometric identification of proteins on membrane detected by Western blotting and lectin blotting. J. Proteome Res. 4, 1391-1396.

Ohtsubo, K., Marth, J.D., 2006. Glycosylation in cellular mechanisms of health and disease. Cell 126, 855-867. 
Parkison, C., Ashizawa, K., McPhie, P., Lin, K.H., Cheng, S.Y., 1991. The monomer of pyruvate kinase, subtype M1, is both a kinase and a cytosolic thyroid hormone binding protein. Biochem. Biophys. Res. Commun. 179, 668-674.

Peter-Katalinic, J., 2005. O-Glycosylation of proteins. Methods Enzymol. 405, 139-171.

Piec, I., Listrat, A., Alliot, J., Chambon, C., Taylor, R.G., Bechet, D., 2005. Differential proteome analysis of aging in rat skeletal muscle. FASEB J. 19, 1143-1145.

Qiu, R., Regnier, F.E., 2005. Use of multidimensional lectin affinity chromatography in differential glycoproteomics. Anal. Chem. 77, 2802-2809.

Renault, V., Thornell, L.E., Eriksson, P.O., Butler-Browne, G., Mouly, V., 2002. Regenerative potential of human skeletal muscle during aging. Aging Cell 1, 132-139.

Schoneich, C., Viner, R.I., Ferrington, D.A., Bigelow, D.J., 1999. Age-related chemical modification of the skeletal muscle sarcoplasmic reticulum Ca-ATPase of the rat. Mech. Ageing Dev. 107, 221-231.

Schwientek, T., Mandel, U., Roth, U., Muller, S., Hanisch, F.G., 2007. A serial lectin approach to the mucin-type $O$-glycoproteome of Drosophila melanogaster S2 cells. Proteomics 7, 3264-3277.
Sharov, V.S., Schoneich, C., 2007. Proteomic approach to aging research. Expert Rev. Proteomics 4, 309-321.

Squier, T.S., Bigelow, D.J., 2000. Protein oxidation and agedependent alterations in calcium homeostasis. Front. Biosci. 5, 504-526.

Stuart, D.I., Levine, M., Muirhead, H., Stammers, D.K., 1979. Crystal structure of cat muscle pyruvate kinase at a resolution of $2.6 \AA$ A. J. Mol. Biol. 134, 109-142.

Towbin, H., Staehelin, T., Gordon, J., 1979. Electrophoretic transfer of proteins from polyacrylamide gels to nitrocellulose sheets: procedure and some applications. Proc. Natl. Acad. Sci. USA 76, 4350-4354.

Varki, A., 2006. Nothing in glycobiology makes sense, except in the light of evolution. Cell 126, 841-845.

Webster, J., Oxley, D., 2005. Peptide mass fingerprinting: protein identification using MALDI-TOF mass spectrometry. Methods Mol. Biol. 310, 227-240.

Wuhrer, M., Catalina, M.I., Deelder, A.M., Hokke, C.H., 2007. Glycoproteomics based on tandem mass spectrometry of glycopeptides. J. Chromatogr. B: Anal. Technol. Biomed. Life Sci. 849, 115-128.

Yan, A., Lennarz, W.J., 2005. Unraveling the mechanism of protein $N$-glycosylation. J. Biol. Chem. 280, 3121-3124. 